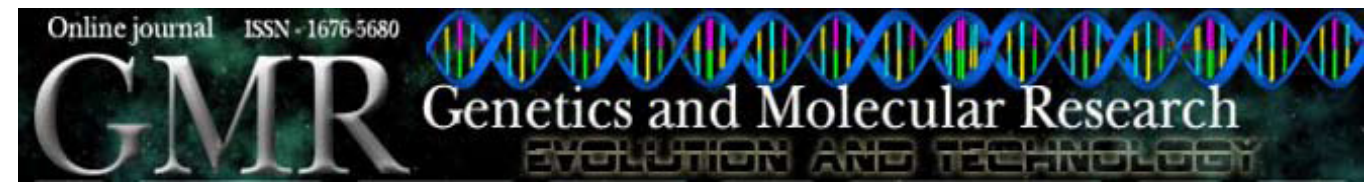

\title{
Lack of effect of bone morphogenetic protein 2 and 4 gene polymorphisms on bone density in postmenopausal Turkish women
}

\author{
Z.S. Özkan' ${ }^{1}$ D. Deveci² ${ }^{2}$ E. Önalan Etem ${ }^{2}$ and H. Yüce ${ }^{2}$ \\ ${ }^{1}$ Kecioren Education and Research Hospital, Department of Gynecology, \\ Ankara, Turkey \\ ${ }^{2}$ Firat University School of Medicine, Department of Genetic, Elazig, Turkey \\ Corresponding author: Z.S. Özkan \\ E-mail: zehrasema@yahoo.com
}

Genet. Mol. Res. 9 (4): 2311-2316 (2010)

Received July 20, 2010

Accepted September 11, 2010

Published November 30, 2010

DOI 10.4238/vol9-4gmr922

\begin{abstract}
We investigated the effect of bone morphogenetic protein 2 and 4 (BMP-2 and -4) gene polymorphisms on bone density in postmenopausal Turkish women with osteoporosis. The frequency of single-nucleotide polymorphisms (SNPs) of BMP2 and -4 genes was analyzed in 101 osteoporotic-postmenopausal women and 52 postmenopausal women with positive bone mineral density scores. We evaluated the frequency of the thymine $\rightarrow$ cytosine nucleotide variation at position 538 for BMP-4 and the transposition of adenine $\rightarrow$ thymine at codon 190 for BMP-2, with PCR. The proportions of genotypes observed for the BMP-2 SNP in the osteoporotic group were AA (47.5\%), AT (39.6\%), TT (12.9\%), and in the non-osteoporotic group they were AA (48.1\%), AT (40.4\%), TT $(11.5 \%)$. The corresponding frequencies for the BMP-4 SNP in the osteoporotic group were TT (30.7\%), TC (45.5\%), CC (23.8\%), and in the non-osteoporotic group they were TT $(26.9 \%)$, TC $(40.4 \%)$, $\mathrm{CC}(32.7 \%)$. There were no significant differences in the frequencies of these genotypes between the patient and control groups. We conclude that genetic variations in BMP-2 and -4 do not substantially
\end{abstract}


contribute to lumbar spine bone mineral density in postmenopausal Turkish women.

Key words: Bone mineral density; Single-nucleotide polymorphism; Bone morphogenetic protein 2 and 4; Genetic association; Osteoporosis

\section{INTRODUCTION}

Osteoporosis is a common disease that affects millions of people throughout the world. The disease is characterized by a reduction of bone mass resulting from a negative balance between bone destruction and bone formation (Baylink et al., 1999). Twin and sibling studies have revealed that the proportion of variance of bone mineral density (BMD) accounted for by genetic factors is around 50-90\% (Flicker et al., 1995; Young et al., 1995). These studies have suggested that the variation in BMD among individuals is largely caused by genetic factors. Advances in knowledge about the genetic basis of osteoporosis are important because they offer the prospect of developing genetic markers for the assessment of fracture risk and the opportunity to identify new targets to design new drugs for the prevention and treatment of osteoporosis (Medici et al., 2006).

Bone morphogenic proteins (BMP) are members of the TGF- $\beta$ superfamily, a large family of growth factors. The BMP subfamily comprises more than 10 proteins, and newer ones are still being discovered. BMP-2 and -4 have $80 \%$ amino acid sequence homology of molecules. BMP-2 and -4 are osteoinductive and regulate osteoblast differentiation (Wang et al., 1990). Follow-up studies demonstrated that genetic variations within BMP-2 were significantly associated with various osteoporotic phenotypes in Icelandic and Danish populations, and thus BMP-2 was reported as a major risk factor for osteoporosis and osteoporotic fractures (Styrkarsdottir et al., 2003). In this study, we investigated whether single-nucleotide polymorphisms (SNPs) in BMP-2 and -4 contribute to lumbar spine BMD in our sample of postmenopausal Turkish osteoporotic and non-osteoporotic women.

\section{MATERIAL AND METHODS}

\section{Subjects}

We analyzed the frequency of SNPs of BMP-2 and -4 genes on 101 osteoporoticmenopausal women (T score on BMD measurement $<-2.5 \mathrm{SD}$ ) and 52 menopausal women with high BMD scores (T score $>+1.0 \mathrm{SD}$ ) who were selected from our menopause outpatient clinic between November 2008 and May 2009. Demographic and lifestyle factors including menopausal period, smoking history, average daily alcohol consumption, and dietary calcium intake were ascertained by lifestyle and food frequency questionnaires (Ireland et al., 1994) completed at baseline. Weight and height were measured at baseline and body mass index (BMI) was calculated. A detailed medical history of subjects was obtained through administration of health questionnaires. Those who had conditions known to affect BMD were excluded from the study. All interviews were conducted by trained interviewers. Informed consent was obtained from all subjects at the time of blood sampling. The study was approved by the Institutional Review Board of Firat University School of Medicine, Elazig. 


\section{BMD measurement}

Areal BMD $\left(\mathrm{g} / \mathrm{cm}^{2}\right)$ at the lumbar spine L2-L4 was measured by dual-energy Xray absorptiometry (DXA) using Lunar PIXI Instrumentation (Madison, WI, USA). Densitometers were calibrated daily. The coefficient of variation for the BMDs of the lumbar spine was $0.52 \%$.

\section{SNP genotyping} methods.

DNA for genetic analysis was extracted from peripheral venous blood by standard

BMP-4 primers used for the polymerase chain reaction (PCR): BMP-4 F: 5'-CCTA ACTGTGCCTAG-3' and BMP-4 R: 5'-CATAACCTCATAAATGTTTATACGG-3' (Mangino et al., 1999).

The PCR amplification conditions were as follows: initial denaturation at $96^{\circ} \mathrm{C}$ for 3 min followed by 35 cycles of $96^{\circ} \mathrm{C}$ for $30 \mathrm{~s}$, followed by $56^{\circ} \mathrm{C}$ for $1 \mathrm{~min}$, and a final extension at $72^{\circ} \mathrm{C}$ for $1 \mathrm{~min}$. Following amplification, 220 bp with PCR product size, $20 \mu \mathrm{L}$ of the product was digested with $H p h \mathrm{I}$ restriction endonuclease (ABgene) at $37^{\circ} \mathrm{C}$. Digested restriction fragments were separated on $2 \%(\mathrm{w} / \mathrm{v})$ agarose (Sigma) gels. A BMP-4 polymorphism site was localized at nucleotide position $538(\mathrm{~T} \rightarrow \mathrm{C})$, resulting in an amino acid change (V147A). After restriction digestion of the PCR products with HphI, 110- and 87-bp band patterns were obtained.

In the BMP-2 gene, a AGA $\rightarrow$ AGT transversion in exon 3, converting arginine to serine was detected at codon 190. For BMP-2 polymorphism detection the amplification refractory mutation system (ARMS) was performed.

BMP-2 primers used for the PCR: forward inner primer (A allele) BMP-2 FI: 5'-CCAACTCGAAATTCCCCGTGACCTGA-3'. Reverse inner primer (T allele) BMP-2 FR: 5'-TTCTGATTCACCAACCTGGTGTCCAAATGA-3'. Forward outer primer (5'-3') BMP-2 OF: 5'-AGAAACGAGTGGGAAAACAACCCGGAGA-3'.

Reverse outer primer (5'-3') BMP-2 OR: 5'-GACACCTTGTTTCTCCTCCAAGTGG GCC-3'.

After PCR, for A allele $174 \mathrm{bp}$ and T allele $234 \mathrm{bp}$ and following the application of two outer primers, a 352-bp product was produced.

PCR was run at $95^{\circ} \mathrm{C}$ for 4 min followed by 30 cycles at $95^{\circ} \mathrm{C}$ for $30 \mathrm{~s}, 60^{\circ} \mathrm{C}$ for $30 \mathrm{~s}$, and $72^{\circ} \mathrm{C}$ for $30 \mathrm{~s}$. A $15-\mu \mathrm{L}$ PCR product was electrophoresed on a $3 \%(\mathrm{w} / \mathrm{v})$ agarose (Sigma) gel (Table 1). BMP-2 SNPs with previously reported variant allele frequencies of $>10 \%$ were selected from the NCBI-SNP [http:// www.ncbi.nlm.nih.gov/SNP] database (Choi et al., 2006).

Table 1. Polymorphisms detected by PCR analysis of BMP-2 and BMP-4 genes.
\begin{tabular}{llcllll}
\hline Gene & Chromosome & Exon & Codon & Polymorphism & Amino acid & Method \\
\hline BMP-4 & $14 \mathrm{q} 22-23$ & 5 & 147 & $\mathrm{~T} \rightarrow \mathrm{C}$ & Valine $\rightarrow$ Alanine & RFLP(Hphl) \\
BMP-2 & $20 \mathrm{p} 12$ & 3 & 190 & $\mathrm{~A} \rightarrow \mathrm{T}$ & Arginine $\rightarrow$ Serine & ARMS \\
\hline
\end{tabular}

$\mathrm{RFLP}=$ restriction fragment length polymorphism; ARMS = amplification refractory mutation system. 


\section{Statistical analysis}

Statistical analyses were performed using SPSS for Windows, version 11.5 (SPSS Inc., Chicago, USA). Stepwise regression analysis was employed on lumbar spine BMD using BMI, age, smoking, alcohol intake, and treatment (placebo or calcium) to identify significant covariates with BMD. A P value of 0.10 or less was required for retention of a covariate in the regression model. Hardy-Weinberg equilibrium was tested for each genotyped SNP using $\chi^{2}$ statistics. To test the effect of each genotyped SNP on lumbar spine BMD, analysis of variance (ANOVA) was performed for groups separately. The independent variable in ANOVA was genotyped, which took on three levels corresponding to the three genotypes observed for each SNP $(1,1 ; 1,2 ; 2,2)$. The proportion of BMD accounted for by each SNP was estimated by the traditional ANOVA $\mathrm{R}^{2}$ value. $\mathrm{P}$ values of $<0.05$ were considered to be significant for all the analyses.

\section{RESULTS}

The mean ages of osteoporotic and non-osteoporotic groups were $57.8 \pm 6.7$ and 53.5 \pm 5.4 years, respectively. The mean menopausal period of the osteoporotic group was $10.2 \pm$ 7.5 years and the non-osteoporotic group was $5.3 \pm 4.5$ years. The mean BMI of the groups were $27.0 \pm 4.5$ and $31.3 \pm 4.7 \mathrm{~kg} / \mathrm{m}^{2}$, respectively. Lumbar BMD of the osteoporotic group was $0.811 \pm 0.086 \mathrm{~g} / \mathrm{cm}^{2}$ and the non-osteoporotic group was $1.362 \pm 0.277 \mathrm{~g} / \mathrm{cm}^{2}$. None of the covariates approached statistical significance $(\mathrm{P}<0.10)$ in the regression model fitting for BMD. The distribution of SNPs and base percentages can be seen in Table 2. The proportions of genotypes observed for the BMP-2 SNP in the osteoporotic group were AA (47.5\%), AT (39.6\%), TT (12.9\%), and in the non-osteoporotic group they were AA (48.1\%), AT (40.4\%), TT $(11.5 \%)$; the corresponding frequencies for the BMP-4 SNP in the osteoporotic group were TT $(30.7 \%)$, TC $(45.5 \%), \mathrm{CC}(23.8 \%)$, and in the non-osteoporotic group they were TT (26.9\%), TC (40.4\%), CC (32.7\%). For BMP-2 in the osteoporotic and non-osteoporotic groups adenine and thymine were observed with $67.3,32.7,68.2$, and $31.8 \%$, respectively. For BMP-4 the percentages in the osteoporotic and non-osteoporotic groups for thymine and cytosine were 53.4, 46.6, 47.1, and 52.9\%, respectively. There were no significant differences between groups in BMP-2 and -4 SNP proportions. But the C allele was observed with a high prevelance in the non-osteoporotic group. None of the SNPs demonstrated a significant association with lumbar spine BMD in either osteoporotic or non-osteoporotic women.

Table 2. BMP-2 and -4 SNPs and base percentages.

\begin{tabular}{lcclcc}
\hline Allele BMP-2 & T score $<-2.5 \mathrm{SD}$ & T score $>+1.0$ SD & Allele BMP-4 & T score $<-2.5$ SD & T score $>+1.0$ SD \\
\hline AA & $48(47.5 \%)$ & $25(48.1 \%)$ & TT & $31(30.7 \%)$ & $14(26.9 \%)$ \\
AT & $40(39.6 \%)$ & $21(40.4 \%)$ & TC & $46(45.5 \%)$ & $21(40.4 \%)$ \\
TT & $13(12.9 \%)$ & $6(11.5 \%)$ & CC & $24(23.8 \%)$ & $17(32.7 \%)$ \\
Total & $101(100 \%)$ & $52(100 \%)$ & Total & $101(100 \%)$ & $52(100 \%)$ \\
Base & T score $<-2.5 \mathrm{SD}$ & T score $>+1.0 \mathrm{SD}$ & Base & T score $<-2.5 \mathrm{SD}$ & T score $>+1.0 \mathrm{SD}$ \\
Adenine & $136(67.3 \%)$ & $71(68.2 \%)$ & Thymine & $108(53.4 \%)$ & $49(47.1 \%)$ \\
Thymine & $66(32.7)$ & $33(31.8 \%)$ & Cytosine & $94(46.6 \%)$ & $55(52.9 \%)$ \\
Total & $202(100 \%)$ & $104(100 \%)$ & Total & $202(100 \%)$ & $104(100 \%)$ \\
\hline
\end{tabular}

$\mathrm{A}=$ adenine; $\mathrm{T}=$ thymine $\mathrm{C}=$ cytosine. 


\section{DISCUSSION}

In the present study, we tested whether polymorphisms in BMP-2 and -4 influence lumbar spine BMD in postmenopausal osteoporotic and postmenopausal Turkish women with high bone mineral density. None of the SNPs, which we tested, reached statistical significance in our sample. These findings indicate that in our population genetic variations in BMP-2 and -4 are unlikely to influence BMD in the lumbar spine. The lack of genetic associations with BMP-2 SNP genotypes in our population contrast with the results found in the Icelandic and Danish populations (Styrkarsdottir et al., 2003) but correlate with Ichikawa et al. (2006). A polymorphism in the BMP-4 gene, affecting the amino acid sequence, studied by Ramesh et al. (2005), showed association with hip bone density, but in our study we did not observe any association between the BMP-4 SNP and lumbar spine BMD. BMD phenotypes are determined by the complex interaction between genes and environment, and we cannot rule out the possibility that genetic variations in BMP-2 and -4 may in fact contribute to BMD variation in other populations.

Insulin-like growth factor-I (IGF-I) is thought to play an important role in skeletal growth and development through its mitogenic and anabolic effects on epiphyseal growth plate chondrocytes. The BMPs have been shown to promote endochondral osteogenesis, and some members of the BMP family, including BMP-2 and BMP-9, have anabolic effects on chondrocyte metabolism. BMPs may modulate the action of IGF-I via the type 1 IGF receptor and/or IGF binding proteins (Takahashi et al., 2007). 2-N, 6-O-sulfated chitosan (26SCS) is a more potent enhancer of BMP-2 bioactivity and induces osteoblastic differentiation in vitro and in vivo by promoting the BMP-2 signaling pathway, suggesting that 26SCS could be used as the synergistic factor of BMP-2 for bone regeneration (Zhou et al., 2009). It has been shown that the fibrillin-microfibril network is essential in the extracellular control of BMP signaling (Sengle et al., 2008). Secreted phosphoprotein-24 (SPP24) increases the rate and magnitude of BMP-2-mediated ectopic bone formation by regulating the local bioavailability of BMP cytokines and its gene contains at least 2 SNPs. SPP24 has an important role in the genetic regulation of bone mass, particularly during periods of BMP-mediated endochondral bone growth, development and fracture healing (Brochmann et al., 2009).

Twenty BMD loci that reached genome-wide significance were 1p31.3 (GPR177), 2p21 (SPTBN1), 3p22 (CTNNB1), 4q21.1 (MEPE), 5q14 (MEF2C), 7p14 (STARD3NL), 7q21.3 (FLJ42280), 11p11.2 (LRP4, ARHGAP1, F2), 11p14.1 (DCDC5), 11p15 (SOX6), 16q24 (FOXL1), 17q21 (HDAC5), 17q12 (CRHR1), 1p36 (ZBTB40), 6q25 (ESR1), 8q24 (TNFRSF11B), 11q13.4 (LRP5), 12q13 (SP7), 13q14 (TNFSF11), and 18q21 (TNFRSF11A) (Rivadeneira et al., 2009). We learned from large-scale collaborative genome-wide meta-analysis that 9 of 150 candidate genes were associated with regulation of BMD, 4 of which also significantly affected risk for fracture. However, most candidate genes had no consistent association with BMD (Richards et al., 2008, 2009). Association studies based on candidate gene polymorphisms and subsequent meta-analyses, and the more recent genome-wide association studies have clearly identified a handful of genes associated with BMD and/or fragility fractures. Among them are genes coding for the LDL-receptor related protein 5 (LRP5), estrogen receptor alpha (ESR1) and osteoprotegerin (OPG; TNFRSf11b). However, the percentage of osteoporosis risk explained by any of these polymorphisms is small, indicating that most genetic risk factors remain to be discovered and/or that interaction with environmental factors needs further consideration (Ferrari, 2008). In this study, we examined only older postmeno- 
pausal women and it is possible that small effects of these SNPs might reduce bone formation by changing the balance of bone turnover to bone loss and this effect would be discernible after many years. Identifying genes that modulate bone mass, strength or bone loss could ultimately provide the basis for a new therapeutic strategy to treat patients with bone loss. These are preliminary data and further studies are needed in other populations of similar and different ethnicity, age and gender to explain the effect on bone mass.

\section{REFERENCES}

Baylink DJ, Strong DD and Mohan S (1999). The diagnosis and treatment of osteoporosis: future prospects. Mol. Med. Today 5: 133-140.

Brochmann EJ, Behnam K and Murray SS (2009). Bone morphogenetic protein-2 activity is regulated by secreted phosphoprotein-24 kd, an extracellular pseudoreceptor, the gene for which maps to a region of the human genome important for bone quality. Metabolism 58: 644-650.

Choi JY, Shin CS, Hong YC and Kang D (2006). Single-nucleotide polymorphisms and haplotypes of bone morphogenetic protein genes and peripheral bone mineral density in young Korean men and women. Calcif. Tissue Int. 78: 203-211.

Ferrari S (2008). Human genetics of osteoporosis. Best Pract. Res. Clin. Endocrinol. Metab. 22: 723-735.

Flicker L, Hopper JL, Rodgers L, Kaymakci B, et al. (1995). Bone density determinants in elderly women: a twin study. J. Bone Miner. Res. 10: 1607-1613.

Ichikawa S, Johnson ML, Koller DL, Lai D, et al. (2006). Polymorphisms in the bone morphogenetic protein 2 (BMP2) gene do not affect bone mineral density in white men or women. Osteoporos. Int. 17: 587-592.

Ireland P, Jolley D, Giles G, O’Dea K, et al. (1994). Development of the Melbourne FFQ: a food frequency questionnaire for use in an Australian prospective study involving an ethnically diverse cohort. Asia Pac. J. Clin. Nutr. 3: 19-31.

Mangino M, Torrente I, De Luca A, Sanchez O, et al. (1999). A single-nucleotide polymorphism in the human bone morphogenetic protein-4 (BMP 4) gene. J. Hum. Genet. 44: 76-77.

Medici M, van Meurs JB, Rivadeneira F, Zhao H, et al. (2006). BMP-2 gene polymorphisms and osteoporosis: the Rotterdam Study. J. Bone Miner. Res. 21: 845-854.

Ramesh Babu L, Wilson SG, Dick IM, Islam FM, et al. (2005). Bone mass effects of a BMP4 gene polymorphism in postmenopausal women. Bone 36: 555-561.

Richards JB, Rivadeneira F, Inouye M, Pastinen TM, et al. (2008). Bone mineral density, osteoporosis, and osteoporotic fractures: a genome-wide association study. Lancet 371: 1505-1512.

Richards JB, Kavvoura FK, Rivadeneira F, Styrkarsdottir U, et al. (2009). Collaborative meta-analysis: associations of 150 candidate genes with osteoporosis and osteoporotic fracture. Ann. Intern. Med. 151: 528-537.

Rivadeneira F, Styrkarsdottir U, Estrada K, Halldorsson BV, et al. (2009). Twenty bone-mineral-density loci identified by large-scale meta-analysis of genome-wide association studies. Nat. Genet. 41: 1199-1206.

Sengle G, Charbonneau NL, Ono RN, Sasaki T, et al. (2008). Targeting of bone morphogenetic protein growth factor complexes to fibrillin. J. Biol. Chem. 283: 13874-13888.

Styrkarsdottir U, Cazier JB, Kong A, Rolfsson O, et al. (2003). Linkage of osteoporosis to chromosome 20p12 and association to BMP2. PLoS Biol. 1: E69.

Takahashi T, Morris EA and Trippel SB (2007). Bone morphogenetic protein-2 and -9 regulate the interaction of insulinlike growth factor-I with growth plate chondrocytes. Int. J. Mol. Med. 20: 53-57.

Wang EA, Rosen V, D’Alessandro JS, Bauduy M, et al. (1990). Recombinant human bone morphogenetic protein induces bone formation. Proc. Natl. Acad. Sci. U. S. A. 87: 2220-2224.

Young D, Hopper JL, Nowson CA, Green RM, et al. (1995). Determinants of bone mass in 10- to 26-year-old females: a twin study. J. Bone Miner. Res. 10: 558-567.

Zhou H, Qian J, Wang J, Yao W, et al. (2009). Enhanced bioactivity of bone morphogenetic protein-2 with low dose of 2-N, 6-O-sulfated chitosan in vitro and in vivo. Biomaterials 30: 1715-1724. 